\title{
Vessel Remodeling after Intima-to-Intima Contact Anastomosis
}

\author{
Hyeonjung Yeo ${ }^{1}$, Hyodong $\mathrm{Kim}^{1}$, Daegu Son ${ }^{2}$, Changbae Hong ${ }^{3}$, Sun Young Kwon ${ }^{4}$ \\ ${ }^{1}$ Department of Plastic and Reconstructive Surgery, Daegu Fatima Hospital, Daegu; ${ }^{2}$ Department of Plastic and Reconstructive Surgery, \\ Keimyung University School of Medicine, Daegu; ${ }^{3}$ College of Physical Education, Keimyung University, Daegu; ${ }^{4}$ Department of Pathology, \\ Keimyung University School of Medicine, Daegu, Korea
}

Background Intima-to-intima microanastomotic vascular remodeling was explored, utilizing a polylactide-caprolactone absorbable vein coupler model (PAVCM), which was designed to simulate a non-absorbable counterpart system with the sole exception of being absorbable.

Methods Six New Zealand white rabbits were used. After transection of the jugular vein, 2 PAVCMs were placed, 1 at each transected end. The stumps were slipped through the PAVCMs, and the venous wall was everted $90^{\circ}$ to achieve intima-to-intima contact. Reanastomosis of the transected jugular vein was performed bilaterally in 3 rabbits. In the other 3 rabbits, the jugular vein $(20 \mathrm{~mm})$ harvested from one side was interpositionally grafted to the jugular vein on the opposite side to ease the anastomotic tension. Patency testing, ultrasonography, and histologic assessments were conducted postoperatively at weeks 2, 4, 12, 16, 22, and 26. Results All anastomotic sites were patent, without stenosis, occlusion, or dilatation. In the histologic sections, immature endothelial regeneration was observed at week 2, which was completed by week 4 . Regeneration of the tunica media was noted at week 12 . Between week 22 and week 26, the tunica media fully regenerated and the coupler dissipated entirely. Conclusions Despite the absence of a coupler to act as an anastomotic buttress, the structure and function of all the vessels appeared normal, even histologically. These outcomes are true milestones in the development of an absorbable vein coupler.

Keywords Anastomosis, surgical / Microsurgery / Vascular surgical procedures
Correspondence: Daegu Son Department of Plastic and Reconstructive Surgery, Keimyung University School of Medicine, 56 Dalseong-ro, Jung-gu, Daegu 41931, Korea

Tel: +82-53-250-7636

Fax: +82-53-255-0632

E-mail: handson@dsmc.or.kr

Received: 25 Aug 2016 • Revised: 5 Dec 2016 • Accepted: 2 Jan 2017

pISSN: 2234-6163 • elSSN: 2234-6171 • https://doi.org/10.5999/aps.2017.44.2.95 • Arch Plast Surg 2017;44:95-100

\section{INTRODUCTION}

To date, microsurgery has evolved through advances in microscopy and in microsurgical instruments. In the future, sutureless vascular anastomoses are expected to be standard, just as intestinal anastomotic devices are today. The prospect of a mechanical device to make microanastomosis easier, faster, and safer was originally presented in 1962 [1]. Such devices ultimately attract- ed the attention of microsurgeons following case reports by Ostrup and Berggren [2] in 1986, leading to the development of the GEM microvascular anastomotic coupler system (Synovis Micro Companies Alliance, Birmingham, AL, USA). The GEM coupler, which is thus far the only system in clinical use, consists of 2 high-density polyethylene rings, each with 6-8 stainless steel pins and alternately placed holes. These pins and holes are eventually interlocked and fixed. Before approximation, each 
segment of a vessel is threaded through a ring and circumferentially everted onto the pins, enabling intima-to-intima contact $[2,3]$. Usage of the GEM system, which has been widely reported worldwide in the last 20 years, yields higher patency rates, lower failure rates, and more predictable thrombotic risk than conventional methods [4-6].

Despite its merits, the GEM system is non-absorbable, thus eliciting a foreign body reaction. Some reports of device migration or extrusion have also been published [7-10]. Anastomotic sites bearing GEM couplers lack normal vascular elasticity, and such devices may exert pressure on vessels or nerves. An absorbable vein coupler would resolve these issues, particularly with respect to the chronicity of non-absorbable materials. In addition, the status of regenerating intima-to-intima anastomotic sites may be monitored more easily with an absorbable coupler in place. Although non-absorbable couplers obscure the anastomotic sites, the vascular segments are held firmly in apposition; therefore, remodeling is not a critical clinical issue. When using an absorbable coupler, the potential consequences of incomplete vascular remodeling (i.e., the absence of layer-by-layer continuity) include aneurysm, leakage, or rupture.

As a prototype, we devised an absorbable vein coupler made of a polylactide-caprolactone (PLCL) conduit, originally designed as an absorbable nerve guide. The jugular veins of rabbits were then used for experimental microvascular anastomoses. The purpose of this study was to examine the resultant process of intima-to-intima microanastomotic vascular remodeling.

\section{METHODS}

\section{Polylactide-caprolactone absorbable vein coupler model} The ring-pin GEM system is designed to fixate both ends of a vessel, everting the walls onto separate rings, which then are ap- proximated for intima-to-intima contact. In order to perform all anastomoses in a manner equivalent to that of the GEM system, a Neurolac tube $2 \mathrm{~mm}$ in diameter (Polyganics BV, Groningen, The Netherlands) was trimmed to a 1-mm length in order to simulate the GEM coupler ring (Fig. 1). Our polylactide-caprolactone absorbable vein coupler model (PAVCM) corresponded to the polyethylene ring of the GEM system, anchoring the everted vessels. However, the sutures (a total of 6) were substituted for the 6 metal pins of the GEM coupler, with the goal of reproducing their effects.

The Neurolac tube is typically used as a biodegradable guide for the repair of nerve defects, replacing autologous nerve grafts. Its PLCL composition is undermined after several weeks [11] and is completely degraded after a year [12]. This substance is transparent, flexible, and soft enough for easy piercing by a suture needle. Once the PAVCM is sufficiently absorbed to loosen the sutures, the anastomotic site is unsupported. The objective of this study was to assess whether Vascular regeneration took place via the PAVCM.

\section{Animal experiments}

All experiments were performed in accordance with the guidelines for animal research stipulated by the National Institutes of Health and the protocol of this study was approved by the Institutional Animal Care and Use Committee (KM-2011-38).

Six New Zealand white rabbits, weighing approximately $3 \mathrm{~kg}$, were anesthetized by an intramuscular injection $(0.7 \mathrm{~mL})$ of a combination of tiletamine hypochloride and zolazepam hypochloride (Zoletil, Virbac, Carros, France) with $0.3 \mathrm{~mL}$ of xylazine (Rompun, Bayer, Leverkusen, Germany), as preparation for surgery in the supine position. A longitudinal neck incision (2-3 cm in length) was made from the midline laterally, followed by an incision of the platysma. The jugular vein was sub-

Fig. 1. Absorbable vein coupler model

(A) Post-microanastomotic view and (B) schematic illustration.

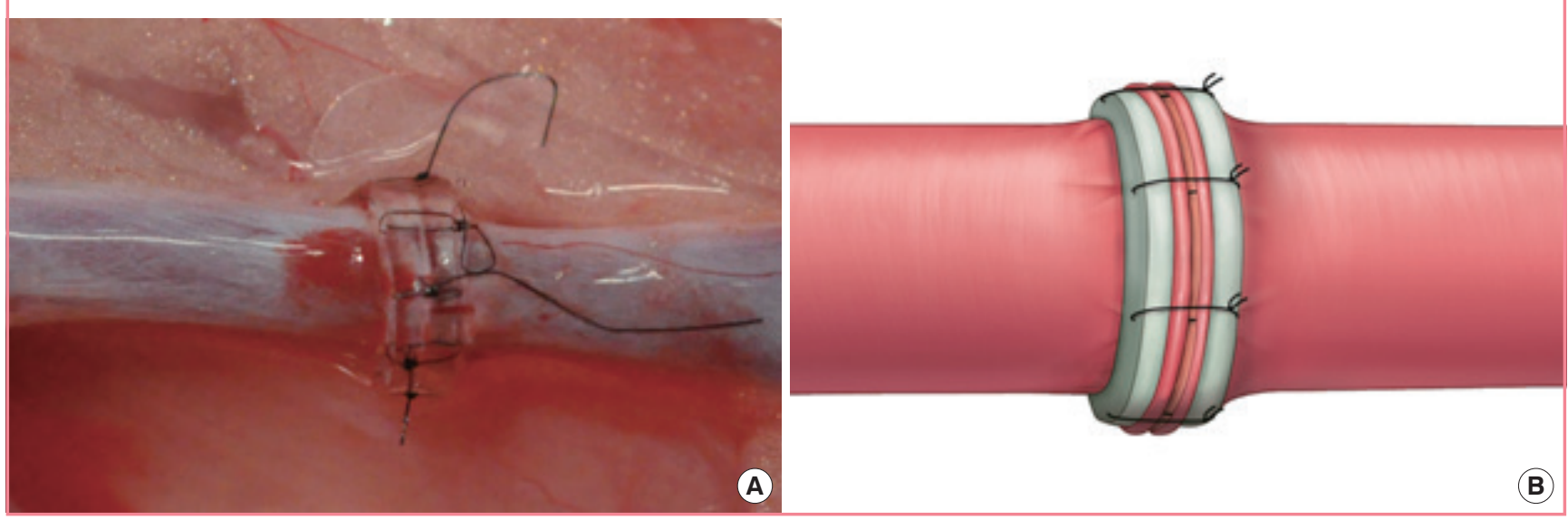


sequently isolated, and a double microvascular clamp was applied. The vein was then transected using microscissors, and each stump was irrigated with heparinized saline before anastomosis. Two PAVCMs were placed, 1 at each transected end; the stumps were slipped through the tubes, the venous wall was everted $90^{\circ}$ (as with the GEM system), and the vein was secured to the tubes with 9-0 black nylon monofilament sutures (Ethilon, Ethicon Inc., Somerville, NJ, USA). Six sutures were regularly placed at $60^{\circ}$ angles, fastening the vessel ends and tubes together. At this point, only the everted intima of both segments made contact (intima-to-intima contact), leaving the vascular lumina free of the suture material (Fig. 1).

Two variations of the experimental surgery were conducted. Reanastomosis of the transected jugular vein was performed bilaterally as above (Fig. 1A) in 3 rabbits. In the other 3, the jugular vein $(20 \mathrm{~mm})$ harvested from one side was interpositionally grafted to the jugular vein on the opposite side, in order to ease the anastomotic tension (Fig. 2A). The same surgeon performed all the anastomotic procedures. Once completed, the incisions were closed in a single layer and the animals were returned to the animal care facility.

\section{Blood flow monitoring and histologic examination}

Immediately after each procedure, the vessels were inspected microscopically for leakage, and patency was confirmed by testing via emptying and refilling. All anastomoses were exposed at weeks $2,4,12,16,22$, and 26 postoperatively ( 1 rabbit for each stage) for patency checks and inspections under loupe magnification. An ultrasonographic examination was performed to detect luminal compromise (i.e., a thrombus, narrowing, or occlusion) or aneurysm development at the anastomotic sites. A 15MHz linear array transducer (iU22, Philips Medical Systems, Bothell, WA, USA), designed specifically for small targets, was used for this purpose. Following ultrasonography, tissue examinations were conducted; $1-\mathrm{cm}$ specimens were removed from both sides of the PAVCMs for $10 \%$ formaldehyde fixation, routine processing, and staining with hematoxylin and eosin. Each rabbit was sacrificed after the patency test, ultrasonographic examination, and biopsy.

\section{RESULTS}

A total of 12 anastomotic sites were examined, including bilateral single anastomoses $(\mathrm{n}=6)$ and dual unilateral anastomoses $(n=6)$. At postoperative week 16 , patency testing and ultrasonography could not be conducted due to an isolated death caused by anesthetic issues. In this case, only the histologic examination was performed.

\section{Macroscopic findings}

Directly after the anastomotic operations, no leakages were observed and patency was regularly demonstrated by testing via

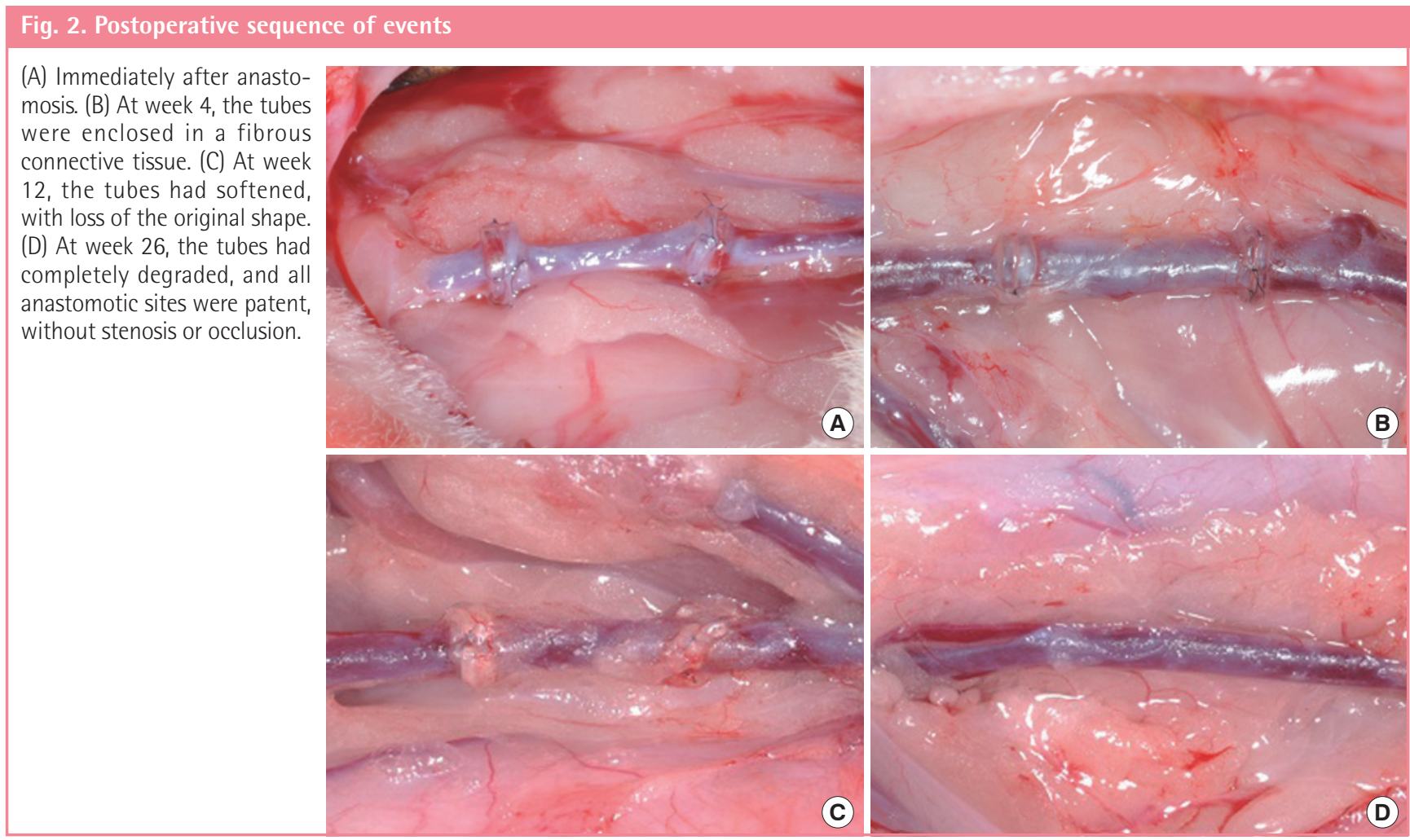


emptying and refilling (Fig. 2A). Loupe magnification at weeks $2,4,12,16,22$, and 26 excluded the presence of thrombi, and testing via emptying and refilling again confirmed vascular patency. By weeks 2 and 4, the tubes were enclosed by a fibrous tissue (Fig. 2B). At week 12, the tubes began to soften (Fig. 2C) and were partially degraded by week 16 , retaining some original form but with less fibrous tissue encasement. Between week 22 and week 26, the tubes were completely degraded, until they were no longer visible (Fig. 2D).

\section{Ultrasonographic findings}

In the ultrasonographic examinations at weeks $2,4,12,16,22$, and 26, no luminal thrombi, narrowing, occlusion, or signs of aneurysm were observed at the anastomotic sites. Patency was demonstrated by normal venous flow through the tubes on Doppler imaging. At week 2, focal luminal narrowing and decreased velocity were noted in 1 tube; otherwise, the overall flow was normal. Two hypoechoic shadows were observed due to the presence of the tubes (Fig. 3).

\section{Histologic findings}

At week 2, the jugular veins showed heavy transmural infiltrates of acute inflammatory cells, with the intima, tunica media, and adventitia involved (Fig. 4A). The inner surfaces appeared relatively smooth; however, clefting (due to folds) was evident at the anastomotic sites (Fig. 4B). The intima was marked by plump endothelial cells, with a loss of intercellular connections.
Regrowth of the medial smooth muscle was not evident, given the ongoing degeneration and necrosis between the tubes and the widespread presence of acute inflammatory cell infiltration. No thrombi or deposits of fibrin were identified.

At week 4, acute inflammation was markedly reduced; however, mild chronic inflammatory cell infiltrates (lymph and plasma cells) and foreign-body giant cells were present, particularly between tubes (Fig. 4C). The inner surfaces were smooth, with continuously regenerative endothelial cell linings (Fig. 4D), but

Fig. 3. Ultrasonographic view at postoperative week 2
Focal luminal narrowing and decreased velocity were present at the
tube; otherwise, a normal overall flow was observed, with 2 hy-
poechoic shadows due to the absorbable vein coupler model.

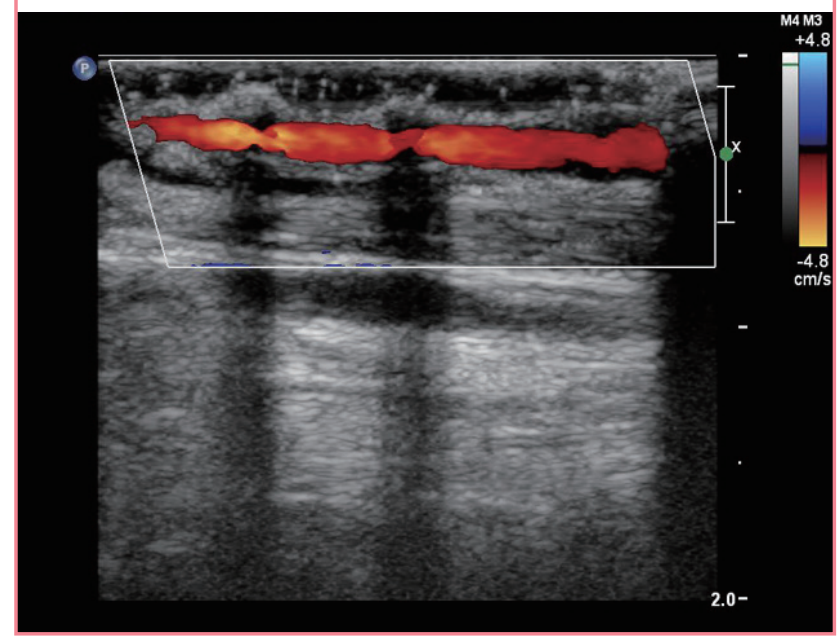

\section{Fig. 4. Timeline of postoperative histologic findings}

The anastomotic site absorbable vein coupler $(\mathrm{N})$ model was placed. Two weeks after anastomosis, the lumen $(\mathrm{L})$ was relatively smooth, except for the anastomotic cleft (due to the fold, black arrow) $(A, \times 10)(B, \times 40)$. Four weeks after anastomosis, the lumen was smooth, with a continuous endothelial cell lining. No visible medial smooth muscle was present at the anastomotic site $(C, \times 10)(D, \times 40)$. Twelve weeks after anastomosis, anastomotic subintimal hyperplasia was observed (black arrow) $(E, \times 10)(F, \times 40)$. Sixteen weeks after anastomosis, the lumen was smooth, and the coupler remained. The full thickness of the medial smooth muscle was restored (black arrow) $(G, \times 10)(H, \times 40)$. All images were obtained using H\&E staining.

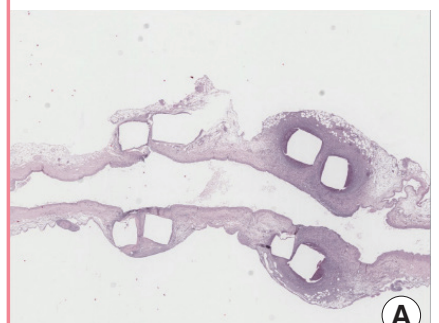

(A)

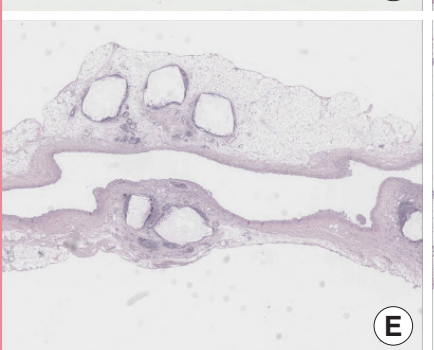

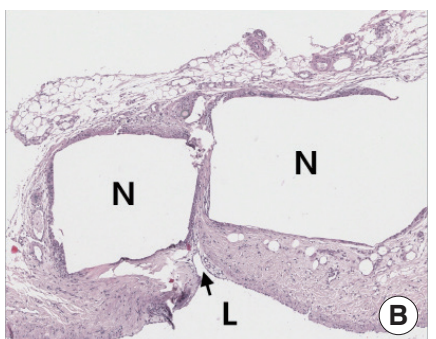

(B)

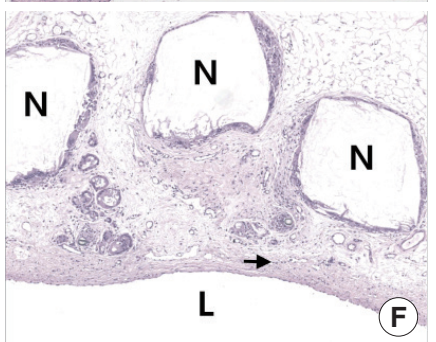

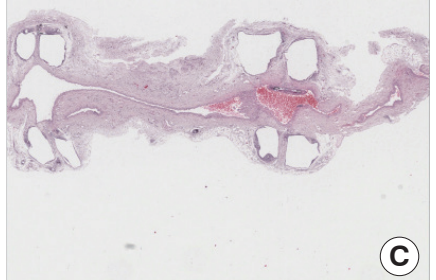

(C)

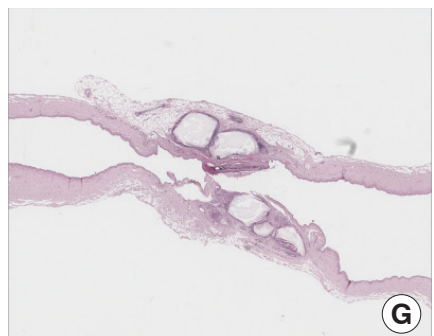

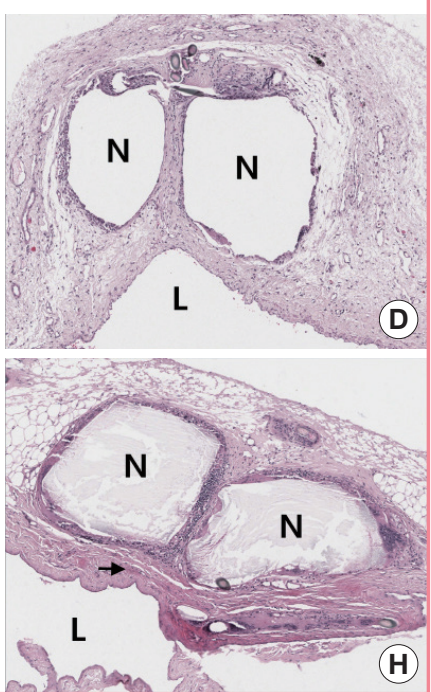


the smooth muscle of the tunica media was still inconspicuous at the points of anastomosis.

At week 12, the tunica media regained some thickness as a result of subintimal hyperplasia (Fig. 4E). Chronic inflammatory cell infiltrates persisted, and foreign-body giant cells were more numerous than at week 4 (Fig. 4F).

At week 16, the luminal surfaces were smooth and the coupler tubes remained present (Fig. 4G). The smooth muscle of the tunica media was restored to full thickness, with a marked reduction in the presence of chronic inflammatory cell infiltrates between the tubes (Fig. 4H). Focal capillary hyperplasia was also observed within the tunica media. Few foreign-body giant cells were present amidst the lingering chronic inflammation.

Between week 22 and week 26, the coupler tubes completely disappeared and chronic inflammation subsided, with no visible infiltrates.

\section{DISCUSSION}

Although great strides have been made in the field of microsurgery, even technically skilled professionals are challenged by microanastomotic procedures. Consequently, committed researchers are diligently pursuing easier, faster, and more accurate surgical methods. In this context, conventional sutures are not only technically difficult, but the sutures alone may also be traumatic to vessel walls. Experimental studies have indicated that tissue necrosis occurs under the compression of suture loops (due to their strangulating effect), and incomplete vascular regeneration (i.e., the persistence of single-cell layers) is possible [13-15]. In other words, vascular regeneration, particularly regeneration of the tunica media, is impeded by the suture material. Sutures may also produce granulomas, generating a mass effect and luminal narrowing [14], and long-term patency may be compromised by a mural thrombus at the suture site.

To overcome the limitations of suturing, sutureless methods have been developed for microanastomosis, including laser welding, electrocoagulation, adhesives (surgical glues), and various mechanical devices. These devices include staplers, such as the vascular closure stapler (VCS) clip (United States Surgical Corp, Norwalk, CT, USA), and ring-pin devices, such as the GEM system (Synovis Micro Companies Alliance). Both types of devices are in use clinically, showing promising results. However, significant atrophy of the tunica media has been detected in animal anastomotic experiments involving vascular couplers. During the regeneration process, the tunica media fails to regenerate, leading to total atrophy, or regenerates minimally, causing compensatory intimal hyperplasia. This happens in conventional anastomoses [15], but it is more striking in conjunction with vascular couplers $[3,16,17]$. The regenerating tunica media forms a thin layer in vessels anchored to the pins, and the vessel walls tend to stretch, becoming quite thin. Mechanical pressure due to a rigid, non-compliant device may also contribute to medial atrophy [18]. The regenerated tissue has less elasticity than a normal vessel, suggesting that mural atrophy may be induced by periodically applied vascular pressure [17,19-21].

In an experimental study by Maxwell et al. [22], the incidence of aneurysms complicating the conventional suturing technique was as high as $48 \%$. Additionally, several case reports of microvascular anastomotic aneurysms have been published [23,24]. Since a true aneurysm is contiguous with the parent vessel, aneurysms stemming from microvascular anastomoses qualify as false aneurysms. The tunica media is absent, and the endothelial layers are thin. In another experiment by Borja-Morant et al. [25], 4 aneurysms developed in 25 arterial anastomoses performed using vascular couplers. Although no clinical reports of aneurysms secondary to vascular couplers have been published to the best of our knowledge, they remain a serious possibility.

The PAVCM proved successful in microvascular anastomosis, as shown by patency, hemodynamic, and histologic assessments. In the histologic preparations, the tunica media fully regenerated, returning to normal thickness at postoperative week 16. At week 22, the coupler tubes degraded completely, with the vascular shape and function still intact. Ultrasonographic examinations confirmed the presence of normal blood flow, without luminal narrowing or aneurysm formation. Hence, there is a strong possibility of this prototype becoming a mainstream device.

We performed different anastomoses in 2 groups to evaluate the impact of anastomotic tension on potential leakage and vascular regenerative outcomes. However, no observable differences were encountered in the comparison of direct anastomoses with interpositional venous grafting. Neither a control group nor a conventional comparator group was included in this study, which was aimed at evaluating intima-to-intima vascular anastomotic remodeling, rather than testing a novel anastomotic technique.

The design of the PAVCM has several advantages. Its semi-rigid construction acts as a stent at the anastomosis site, preventing the back-walling of sutures during anastomosis; postoperatively, it prevents vascular luminal collapse and reduces the risk of stenosis. Additionally, each vascular stump is completely everted, permitting complete visualization of the 2 open channels. The thrombogenic effect of adventitial movement into the suture sites is thereby prevented. Finally, no suture material is anchored on the luminal side, dramatically reducing the risk of a mural thrombus or foreign body reaction. Given these noteworthy 
properties, the PAVCM may supersede conventional venous anastomosis in the future.

\section{REFERENCES}

1. Nakayama K, Yamamoto K, Tamiya T. A new simple apparatus for anastomosis of small vessels: preliminary report. J Int Coll Surg 1962;38:12-26.

2. Ostrup LT, Berggren A. The UNILINK instrument system for fast and safe microvascular anastomosis. Ann Plast Surg 1986;17:521-5.

3. Berggren A, Ostrup LT, Lidman D. Mechanical anastomosis of small arteries and veins with the unilink apparatus: a histologic and scanning electron microscopic study. Plast Reconstr Surg 1987;80:274-83.

4. Ardehali B, Morritt AN, Jain A. Systematic review: anastomotic microvascular device. J Plast Reconstr Aesthet Surg 2014;67:752-5.

5. Yap LH, Constantinides J, Butler CE. Venous thrombosis in coupled versus sutured microvascular anastomoses. Ann Plast Surg 2006;57:666-9.

6. Denk MJ, Longaker MT, Basner AL, et al. Microsurgical reconstruction of the lower extremity using the $3 \mathrm{M}$ microvascular coupling device in venous anastomoses. Ann Plast Surg 1995;35:601-6.

7. Kubo T, Kitamura N, Onoda M, et al. Exposure of a microvascular anastomotic coupler in head and neck reconstruction: a case report. Eplasty 2014;14:e18.

8. Zomerlei TA, Komorowska-Timek E. Venous coupler migration: a near miss. Microsurgery 2014;34:331-2.

9. Hong C, Yeo H, Son D. Vascular remodeling with a microvascular anastomotic coupler system: a case report. Arch Reconstr Microsurg 2015;24:20-3.

10. Kind GM, Buncke GM, Buncke HJ. Foreign-body sensation following $3 \mathrm{M}$ coupler use in the hand. Ann Plast Surg 1996;37:418-21.

11. Bertleff MJ, Meek MF, Nicolai JP. A prospective clinical evaluation of biodegradable neurolac nerve guides for sensory nerve repair in the hand. J Hand Surg Am 2005;30: 513-8.

12. Den Dunnen WF, Meek MF, Robinson PH, et al. Peripheral nerve regeneration through $\mathrm{P}(\mathrm{DLLA}$-epsilon-CL) nerve guides. J Mater Sci Mater Med 1998;9:811-4.

13. Morecraft R, Blair WF, Chang L. Histopathology of microvenous repair. Microsurgery 1985;6:219-28.

14. Chow SP. The histopathology of microvascular anastomosis: a study of the incidence of various tissue changes. Microsurgery 1983;4:5-9.

15. Daniel RK, Lidman D. The normal healing process of microvascular anastomoses. Scand J Plast Reconstr Surg 1981; 15:103-10.

16. Li YN, Wood MB. End-to-side-anastomosis in the dog using the $3 \mathrm{M}$ precise microvascular anastomotic system: a comparative study. J Reconstr Microsurg 1991;7:345-50.

17. Ragnarsson R, Berggren A, Ostrup LT. Long term evaluation of the unilink anastomotic system: a study with light and scanning electron microscopy. Scand J Plast Reconstr Surg Hand Surg 1992;26:167-71.

18. Blair WF, Morecraft RJ, Steyers CM, et al. A microvascular anastomotic device: Part II. A histologic study in arteries and veins. Microsurgery 1989;10:29-39.

19. Daniel RK, Olding M. An absorbable anastomotic device for microvascular surgery: experimental studies. Plast Reconstr Surg 1984;74:329-36.

20. Qu W, Muneshige H, Ikuta Y. An absorbable pinned-ring device for microvascular anastomosis of vein grafts: exprerimental stuies. Microsurgery 1999;19:128-34.

21. Joji S, Muneshige H, Ikuta Y. Experimental study of mechanical microvascular anastomosis with new biodegradable ring device. Br J Plast Surg 1999;52:559-64.

22. Maxwell GP, Szabo Z, Buncke HJ Jr. Aneurysms after microvascular anastomoses: incidence and pathogenesis in experimental animals. Plast Reconstr Surg 1979;63:824-9.

23. Lykoudis EG, Papanikolaou GE, Katsikeris NF. Microvascular anastomotic aneurysms in the clinical setting: case report and review of the literature. Microsurgery 2009;29: 293-8.

24. Ceulemans P, Van Landuyt K, Hamdi M, et al. Complete survival of a free flap after early pseudoaneurysm formation and pedicle thrombosis. Ann Plast Surg 2001;47:332-5.

25. Borja-Morant A, Navarro-Vila C, Cuesta-Gil M, et al. Experimental evaluation and clinical use in the head and neck of a 3M/Precise microvascular anastomotic device. J Craniomaxillofac Surg 1995;23:305-11. 